\title{
Neuronal pentraxin II is highly upregulated in Parkinson's disease and a novel component of Lewy bodies
}

\author{
Linda B. Moran · Lorraine Hickey · Gregory J. Michael • Maria Derkacs • \\ Lynne M. Christian • Michail E. Kalaitzakis · Ronald K. B. Pearce • \\ Manuel B. Graeber
}

Received: 14 September 2007 / Revised: 5 October 2007 / Accepted: 6 October 2007 / Published online: 7 November 2007

(C) Springer-Verlag 2007

\begin{abstract}
Neuronal pentraxin II (NPTX2) is the most highly upregulated gene in the Parkinsonian substantia nigra based on our whole genome expression profiling results. We show here that it is a novel component of Lewy bodies and Lewy neurites in sporadic Parkinson's disease (PD). NPTX2 is also known as the neuronal activity-regulated protein (Narp), which is secreted and involved in long-term neuronal plasticity. Narp further regulates AMPA receptors which have been found to mediate highly selective non-apoptotic cell death of dopaminergic neurons. NPTX2/Narp is found in close association with alpha-synuclein aggregates in both substantia nigra and cerebral cortex in PD but unlike alpha-synuclein gene expression, which is down-regulated in the Parkinsonian nigra, NPTX2 could represent a driver of the disease process. In view of its profound $(>800 \%)$ upregulation and its established role in synaptic plasticity as well as dopaminergic nerve cell death, NPTX2 is a very interesting novel player which is
\end{abstract}

This work was supported by the Parkinson's Disease Society of the UK.

Electronic supplementary material The online version of this article (doi:10.1007/s00401-007-0309-3) contains supplementary material, which is available to authorized users.

L. B. Moran · L. Hickey · M. Derkacs · L. M. Christian .

M. E. Kalaitzakis · R. K. B. Pearce · M. B. Graeber $(\square)$

Imperial College London and Hammersmith Hospitals Trust,

University Department of Neuropathology,

Charing Cross campus, Fulham Palace Road,

London W6 8RF, UK

e-mail: manuel@graeber.net

G. J. Michael

Neuroscience Centre, Institute of Cell and Molecular Science, Queen Mary, University of London, London E1 2AT, UK likely to be involved in the pathway dysregulation which underlies PD.

Keywords AMPA receptors · Cell death · Dementia . Expresssion profiling $\cdot$ Orexin/hypocretin system

\section{Introduction}

Neuronal pentraxins constitute a family of proteins that are homologous to C-reactive and acute-phase proteins in the immune system and are thought to be involved in activity-dependent synaptic plasticity $[1,11,32]$. The neuronal pentraxins (NPTX1, NPTX2) and the neuronal pentraxin receptor were identified as synaptic proteins that bind to affinity columns of the snake venom toxin, taipoxin which presynaptically blocks neurotransmission, and the luminal calcium-binding protein TCBP49 [15]. NPTX2 [14] is also known as neuronal activity-regulated pentraxin (Narp), which was first identified as an immediate early gene responding to the induction of seizures in rat hippocampus [31]. The expression of this protein is regulated by synaptic activity and it induces the formation of new excitatory synapses and the regulation of alphaamino-3-hydroxy-5-methyl-4-isoxazolepropionic acid (AMPA)-type glutamate receptor clustering at established synapses [7, 22, 23]. Importantly, dopaminergic neurons are selectively susceptible to excitotoxicity mediated via AMPA receptor activation with the resulting cell death being non-apoptotic [5].

A second line of research that makes NPTX2 a highly interesting gene in the context of Parkinson's disease (PD) research concerns its expression in hypothalamic nerve cells that co-express hypocretin/orexin [3] and which are prodigiously affected by the neurodegeneration in $\mathrm{PD}$. 
These nerve cells have been implicated in the sleep disturbances that are often found in PD patients [9, 30].

Using a whole genome expression dataset of the human Parkinsonian nigra [19], we have identified NPTX2 as the most highly upregulated gene in a cohort of sporadic PD cases. Here we demonstrate that NPTX2 labels Lewy bodies in paraffin sections and that there is dysregulation of NPTX2 also in PD frontal cortex.

\section{Materials and methods}

Tissue samples from human $\mathrm{SN}$ and frontal cortex

Some of the sporadic PD cases used in this study and several controls have been described previously $[6,19$, 20]. Information on the cases employed for this project is provided in Supplemental Table. Brain tissue was obtained from the UK Parkinson's Disease Society Brain Bank at Imperial College London. The diagnostic work was performed in the Department of Neuropathology following international neuropathological consensus criteria for PD (http://www.ICDNS.org) which require use of alpha-synuclein as a molecular diagnostic marker. Ethics committee approval was available for all human tissue work of this study. For the dissection of the substantia nigra tissue (medial and lateral nigra were prepared separately), the portion of the midbrain containing the substantia nigra was separated from the brainstem distal to the mamillary bodies ( $45^{\circ}$ angle cut) at the level of the third cranial nerve and the superior colliculi. A slice approximately $5 \mathrm{~mm}$ in depth was prepared containing both $\mathrm{SN}$ and the cross-sectioned red nucleus at the level of its greatest diameter. Then, the SN was dissected from the surrounding nuclei and the pars reticulata. Once the $\mathrm{SN}$ was isolated, it was divided into two portions, the medial SN and the lateral SN. In all cases where frontal lobe was used, the superior frontal gyrus was sampled at the same level.

Quantitative real-time polymerase chain reaction

Total RNA was extracted from PD and control cases and transcribed using the RETROscript ${ }^{\circledR}$ kit (Applied Biosystems, Warrington, UK) following the manufacturer's protocol. Primers for the internal control genes were as before [19]. Predesigned Taqman primers for NPTX2 (Hs00383983_m1) were purchased (Applied Biosystems) and Taqman mastermix (Applied Biosystems) was used. Each reaction was run in triplicate using an ABI PRISM ${ }^{\mathrm{TM}}$ 7700 Sequence Detector (Applied Biosystems). For every gene tested, a negative control was run without cDNA template. Data analysis for real-time PCR (DART-PCR
Version 1.0; [25]) was used to determine relative differences in NPTX2 mRNA expression between PD cases and controls (also see Supplemental Figure 1).

In situ hybridization (ISH)

ISH was performed using radioactive oligonucleotide probes as described previously [18, 29] Oligonucleotides were designed using NetPrimer software (Premier, BioSoft International, http://www.premierbiosoft.com). Two oligonucleotides complementary to NPTX2 mRNA at nts 899932 (AGCCACAGGCAGATGGTGAAGGCGTACAGC TCAG-probe A) and nts 1364-1397 (TCGACGTTATT GTCCACCCACGG-GATGATGTTGC-probe B) were synthesized (Sigma Genosys, Poole, UK). Oligonucleotides were radioactively end-labelled with ${ }^{35} \mathrm{~S}$-dATP (PerkinElmer, UK) using terminal deoxynucleotidyl transferase (Promega, UK).

Snap frozen, unfixed post-mortem tissue blocks of the frontal cortex and SN were cut on a cryostat $(12 \mu \mathrm{m})$, collected onto Superfrost Plus slides (VWR) and stored at $-80^{\circ} \mathrm{C}$. Before use, tissue sections were fixed for $5 \mathrm{~min}$ in fresh $4 \%$ paraformaldehyde in $0.1 \mathrm{M}$ phosphate buffer ( $\mathrm{pH}$ 7.4). Pre-hybridisation treatment included acetylation in $0.25 \mathrm{M}$ acetic anhydride $/ 0.1 \mathrm{M}$ triethanolamine, dehydration in graded alcohol (70-100\% ethanols) and delipidation in $100 \%$ chloroform. Hybridisation with the oligonucleotide probe was performed overnight at $37^{\circ} \mathrm{C}$. Post-hybridisation sections were washed in standard saline citrate solutions with increasing stringencies. The sections were dehydrated rapidly through graded alcohols (70-100\% ethanol), air-dried, dipped in autoradiographic emulsion (LM1, GE Healthcare, Amersham, UK) and exposed for 5-6 weeks. The autoradiographic emulsion was developed, sections were counterstained with toluidine blue (VWR) for Nissl substance and mounted for visualisation using bright field microscopy for the counterstain and epi-polarized illumination for the silver grains of the autoradiographic emulsion. Control slides run to check for specificity of hybridization included competition with an excess of oligonucleotide (250 times) and the use of two probes to different portions of the mRNA of interest which produced identical patterns of signal. Black and white photographs of emulsion-coated sections were taken using a microscope provided with a Hamamatsu C4742-95 digital camera (Herrsching, Germany) and HiPic software (Herrsching) to capture images. Digital colour images were captured with a Retiga 1300 monochrome 12-bit camera using a color option provided by a RGB-HM-S filter (QImaging, Burnaby, BC, Canada) and QCapture 1.1.6 software (QImaging). Adobe Photoshop was used to assemble images. 
Fig. 1 Neuronal pentraxin II (NPTX2) is strongly upregulated in substantia nigra and cerebral cortex of PD patients. a NPTX2 immunocytochemistry demonstrating specific staining of Lewy bodies in a dopaminergic neuron. b Western blotting confirms specificity of the antiNPTX2 antibody. $\mathbf{c}$ qRT-PCR shows highly increased levels of NPTX2 mRNA in substantia nigra and, to a lesser extent, in frontal cortex. The arbitrary units along the ordinate represent relative fold changes [25].

The control value is 1 ; error bars indicate SEM. d, e In situ hybridisation demonstrates NPTX2 mRNA expression in nerve cells as well as glia in both substantia nigra and frontal cortex. Labelling is also found over non-pigmented neurons (arrow in $\mathbf{d}, \mathrm{SN}$ ). The arrows in e mark glial cells (cortex). In addition, there is autoradiographic signal in the neuropil (asterisks) which would be in keeping with the presumed dendritic expression of NPTX2 mRNA. f NPTX2 immunocytochemistry of cortical neuropil reveals occasional intercellular patches of "arborised" labelling also possibly suggestive of a dendritic localisation (asterisks). The arrow points to a NPTX2

immunoreactive glial cell. Counterstaining in $\mathbf{a}$ and $\mathbf{f}$, haemalum, and toluidine blue in d. Scale bars: $20 \mu \mathrm{m}(\mathbf{a}, \mathbf{f})$, $50 \mu \mathrm{m}(\mathbf{d})$, and $100 \mu \mathrm{m}(\mathbf{e})$
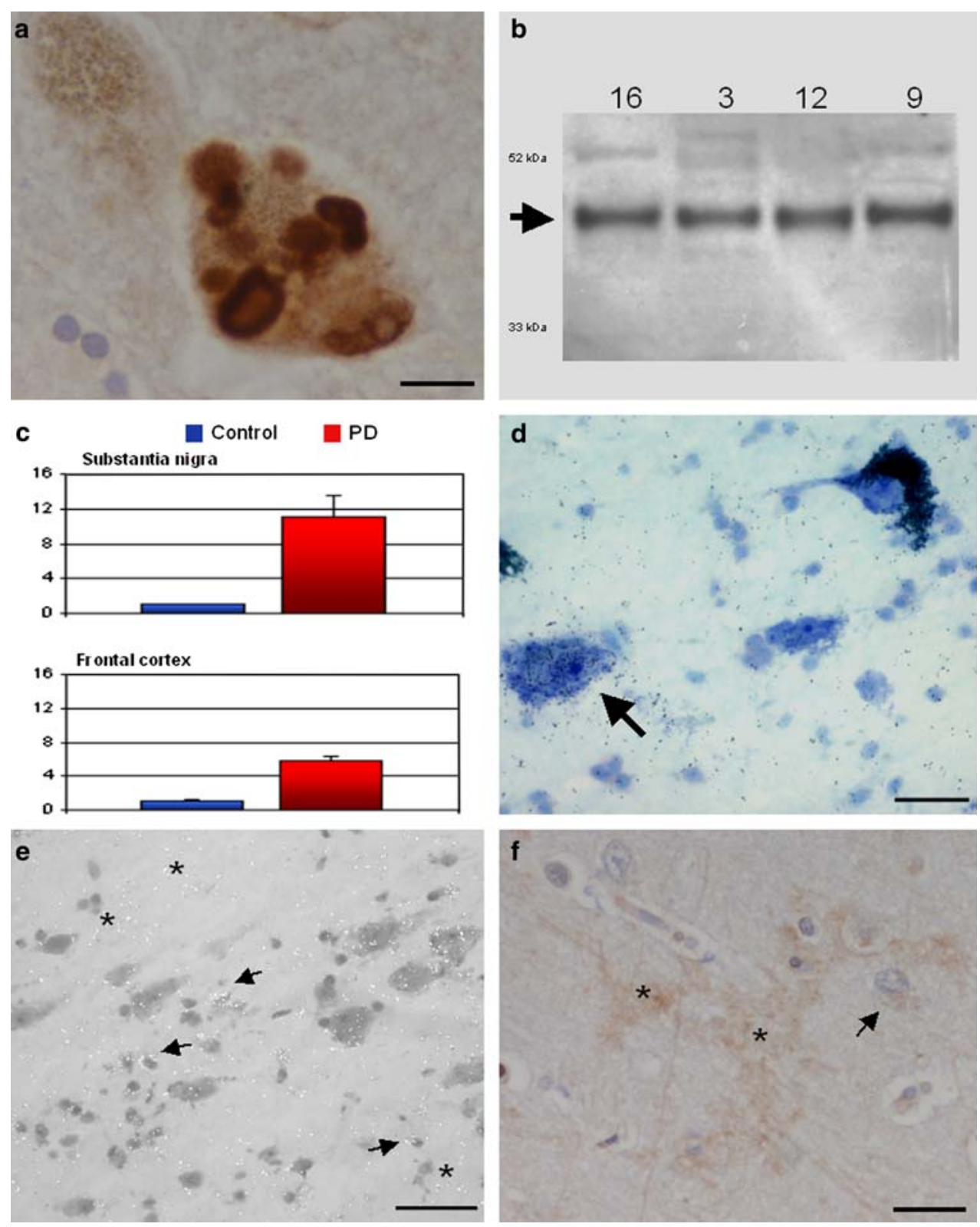

\section{Immunohistochemistry}

Paraffin embedded tissue sections $(8 \mu \mathrm{m})$ were used for all stainings. No antigen retrieval was required for NPTX2 whereas for alpha-synuclein (SNCA) antibody staining sections were treated with $80 \%$ formic acid for $60 \mathrm{~min}$ [24]. Adjacent sections from SN were stained with goat polyclonal anti-NPTX2 antibody (NP2 N-20, sc-12125; Santa Cruz Biotechnology Inc.; 1:25) and mouse monoclonal anti-SNCA (BD Transduction Laboratories, catalogue number $610787 ; 1: 1,000)$ overnight $\left(4^{\circ} \mathrm{C}\right)$. Secondary antibodies used were gG-HRP (sc-2020; Santa Cruz Biotechnology Inc.; 1:100) and biotinylated horse anti-mouse (B-2000; Vector Laboratories, 1:100), respectively. The ABC kit (Elite 6100, Vector Laboratories) was used for visualisation using 3,3'-diaminobenzidine tetrahydrochloride $(0.02 \%$; Sigma, UK). Sections were counter-stained in Mayer's haemalum.

For fluorescence microscopy, anti-SNCA $(1: 1,000)$ and anti-NPTX2 (1:25) were added to the sections sequentially (each was incubated overnight at $4{ }^{\circ} \mathrm{C}$ ). For the detection of the primary antibodies, sections were rinsed in $0.1 \mathrm{M}$ Tris buffered saline (TBS) and then incubated with either the goat anti-rabbit IgG tagged with Alexa Fluor 546 (red) for $1 \mathrm{~h}$ at room temperature or the biotinylated anti-mouse antibody (Vector Laboratories 1:100) for 1 hour followed by incubation with streptavidin Alex Fluor 488 (green) for $1 \mathrm{~h}$ at room temperature. Finally, tissue sections were rinsed in TBS and incubated in sudan black for $10 \mathrm{~min}$ before mounting in $80 \%$ Tris-buffered glycerol. 

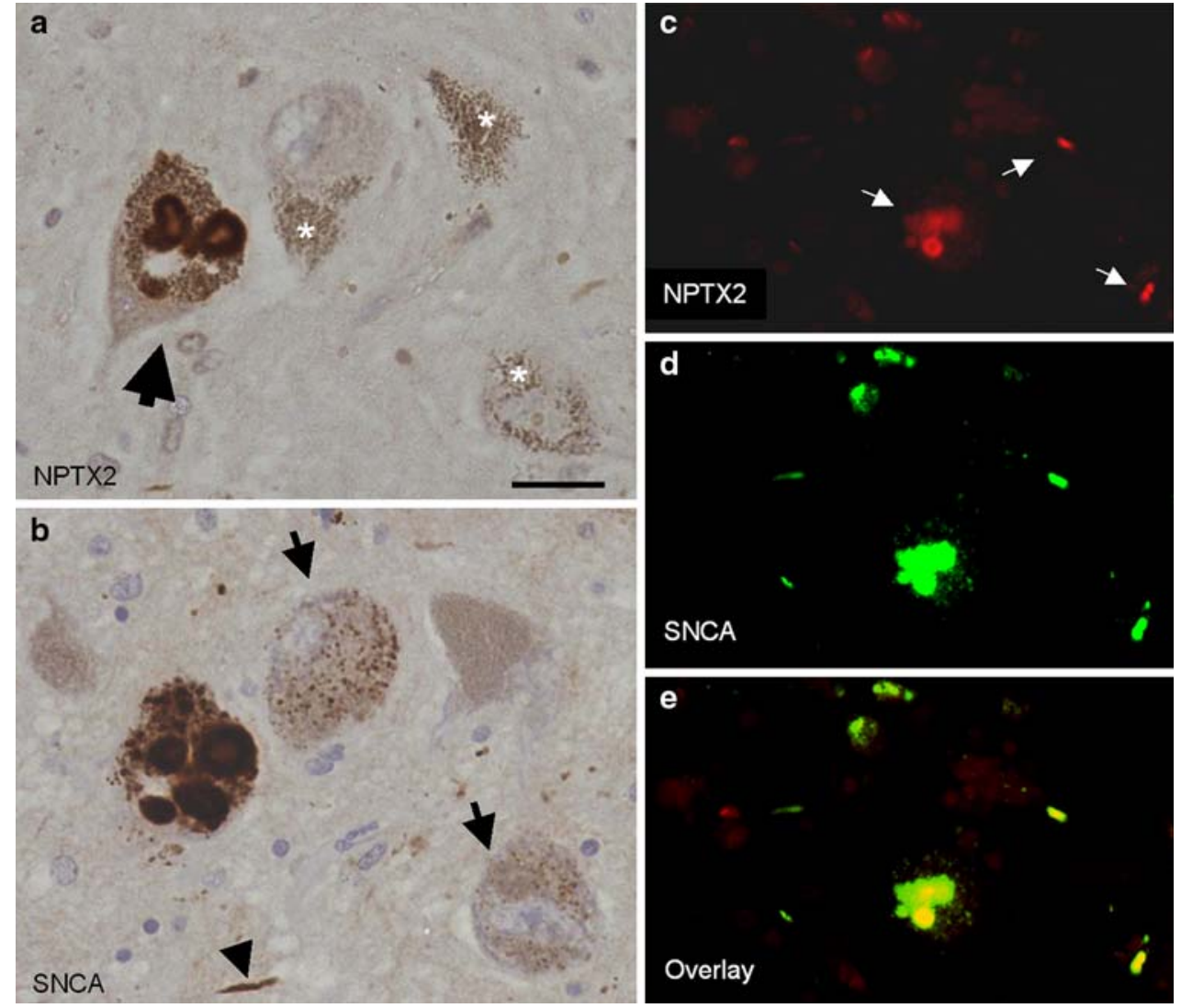

Fig. 2 Co-localisation of NPTX2 and alpha-synuclein. a, b NPTX2 and alpha-synuclein immunolabelling of adjacent tissue sections demonstrates significant but not complete overlap between the two proteins. The large arrow in a denotes the same Lewy body-containing dopaminergic nigral neuron. Small granular cytoplasmic deposits showing alpha-synuclein immunoreactivity in some nerve cells (arrows in b) are NPTX2 negative. These aggresomes are sometimes difficult to distinguish from neuromelanin granules based on staining

\section{Western blotting}

Snap frozen midbrain tissue samples containing SN from PD and control cases were homogenized with RIPA buffer ( $\mathrm{pH} 8$ ), heated to $70^{\circ} \mathrm{C}$ for $10 \mathrm{~min}$ and subsequently centrifuged $(13,000 \mathrm{rpm}, 10 \mathrm{~min})$. The protein supernatant was removed and the concentration of protein determined (BIO-RAD protein reagent; BIO-RAD, UK) using a spectrophotometer. Denaturing gels were run using $30 \mu \mathrm{g}$ of protein from each sample in $19.5 \mu \mathrm{l}$ RIPA buffer, $3 \mu 1 \beta$-mercaptoethanol and $7.5 \mu 1$ lithium dodecyl sulphate sample buffer (Invitrogen, UK). NuPAGE Bis-Tris 4-12\% polyacrylamide gels (Invitrogen) were run in NuPage SDS running buffer (Invitrogen). Gels were blotted onto nitrocellulose membranes (Invitrogen), immersed in NuPage LDS transfer buffer (Invitrogen) in a cold room $\left(4^{\circ} \mathrm{C}\right)$ overnight applying $30 \mathrm{~V}$. Successful protein transfer was verified by staining the membrane in Ponceau S (VWR, UK) for $10 \mathrm{~min}$. alone but their cytoplasmic distribution is also different from the latter. In contrast, Lewy neurites are double-labelled (arrow head in b). Neuromelanin (asterisks in a) is unstained. c, d, e Dual immunofluorecence demonstrates co-localisation of neuronal pentraxin II $(\mathrm{red}, \mathbf{c})$ and alpha-synuclein (green, d). Double-labelled structures (arrows in c) light up in yellow in the overlay (e). Scale bar: $40 \mu \mathrm{m}(\mathbf{a}, \mathbf{b})$, and $80 \mu \mathrm{m}$ (c-e), respectively

Prior to overnight incubation in polyclonal goat antibody to NPTX2 (N-20), membranes were washed in phosphate buffered saline (PBS) containing 0.1\% Tween 20 and subsequently rinsed in PBS/0.1\% Tween 20 containing $5 \%$ skimmed milk (blocking step) for $30 \mathrm{~min}$. Incubation with the secondary antibody coupled to horseradish peroxidase $(1: 5,000$; Vector Labs, UK) was carried out for $1 \mathrm{~h}$ at room temperature. Visualization of antibody binding using enhanced chemilluminescence (ECL; Amersham Biosciences, UK) was performed according to the manufacturer's instructions.

\section{Semi-quantitative ratings}

The NPTX2 labelled SN sections were examined by two independent observers blinded to the identity of the respective cases. The occurrence of NPTX2 immunoreactive profiles in the SN was scored for the overall burden of NPTX2 immunoreactive deposits using a seven-point scale ranging 
from $0-0.5$ to 3 (absent to severe). Correlation analysis (Spearman; Sigmatstat 2.03) was conducted within the PD cohort for NPTX2 mRNA expression (log 2 microarray data) and the corresponding semi-quantitative immunocytochemical results.

\section{Results}

NPTX2 is a novel component of Lewy bodies

We demonstrate here that NPTX2 protein in the Parkinsonian substantia nigra localizes to Lewy bodies, Lewy neurites and some glial cells. NPTX2 is the most upregulated gene in our microarray data set [19]. It shows the greatest difference between disease and control nigrae (probe 213479_at, $P=4.60051 \mathrm{E}-07$, differential expression, $\mathrm{DE}_{\log 2}, 3.0036217$ which is equivalent to $>800 \%$ ). Many neurons show more than one NPTX2 immunoreactive LB (Fig. 1a). Western blotting confirmed specificity of the antibody revealing a band, which corresponds to the NPTX2 monomer (47 kDa; Fig. 1b). Very strong upregulation of NPTX2 mRNA expression in PD was confirmed by means of quantitative real time-PCR (Fig. 1c).

\section{Localisation of NPTX2 and SNCA protein}

In situ hybridisation revealed expression of NPTX2 mRNA in nerve cells as well as glia in both SN and frontal cortex, respectively (Fig. 1d, e). NPTX2 immunoreactive LBs were also observed in the frontal cortex and there were ramified ("arborised") NPTX2 positive profiles of cell processes in the neuropil (Fig. 1f). Analysis of adjacent tissue sections demonstrated co-localisation of NPTX2 and SNCA in the same nerve cells (Fig. 2a, b). This was confirmed by dual immunofluorescence (Fig. 2c-e). Overall, NPTX2 immunoreactive neuronal deposits mapped very closely to alpha-synuclein aggregates, both cellularly and subcellulary, and NPTX2 was also observed in incidental Lewy bodies (for a summary of cases used see Supplemental Table of Electronic Supplementary Material). In contrast, no NPTX2 immunoreactive intracellular aggregates were observed in two cases of tauopathy, i.e. progressive supranuclear palsy, where alpha-synuclein deposits are also not found. However, not all SNCA immunoreactive profiles were NPTX2 positive, i.e. the number of SNCA labelled nerve cells outnumbered that of NPTX2 stained ones (about 2-3:1). In addition, in some nerve cells, SNCA immunopositivity was observed as small granular cytoplasmic deposits ("aggresomes") that lacked NPTX2 immunoreactivity (Fig. 2a, b). However, NPTX2 immunoepitopes were found to decorate the halo of LBs more distinctly than alpha-synuclein (Fig. 3). Strongly NPTX2 immunoreactive Lewy neurites (Fig. 3) were also found. Cases with high levels of NPTX2 protein expression showed a larger alphasynuclein burden. We estimate that about one-third of the alpha-synuclein immunoreactive protein deposits also stain for NPTX2. Labelling of normal appearing axons by NPTX2 was widespread in both PD (Fig. 3) and control brains. Glial cytoplasmic (Papp-Lantos) inclusions of multiple system atrophy (MSA) were NPTX2 positive (not shown) and in PD a sizeable number of glial cells expressed NPTX2 protein that appeared to be SNCA negative (Fig. 3). Correlation analysis for NPTX2 microarray expression data and the corresponding semi-quantitative immunocytochemical case ratings showed a significant correlation $(r=0.970, P<0.001$; Table 1$)$. However, there was no association between NPTX2 expression and duration of the disease.

\section{Discussion}

The most significant finding of this study is the striking presence of NPTX2 in classical Lewy bodies and in Lewy neurites. Experimental studies have demonstrated that Narp, the rat homologue of NPTX2, is important in neuronal development, promoting neuronal migration, synapse formation [1, 8] and neurite outgrowth [31]. Studies in neuronal pentraxin I and neuronal pentraxin II knockout mice have demonstrated a role in early synaptic refinements in the retina and dorsal lateral geniculate nucleus during development [1]. It has also been suggested that Narp's role in synaptic plasticity may underlie the long-lasting aversive effects of drug withdrawal $[10,26]$. These functions of the NPTX2 gene product and its dendritic expression in the cerebral cortex [17] are of special interest in relation to our finding that NPTX2 immunoreactivity was also occasionally present in the cortical neuropil where it had a ramified appearance reminiscent of dendritic arbors.

There is limited knowledge on the NPTX2 pathway (Fig. 4). Known physiological functions of NPTX2 include a role in the induction of AMPA receptor clustering [7]. NPTX2, NPTX1 and the GRIA3 subunit of AMPA receptors are all upregulated in our dataset. All other AMPA subunits and the receptor for NPTX2, NPTXR are downregulated. Two additional genes whose functions may be associated with AMPA receptors in dopaminergic neurons are also highly dysregulated in PD, NSF ( $N$-ethylmaleimide sensitive fusion protein; 202395_at, $P=1.18252 \mathrm{E}-05$, $\mathrm{DE}_{\log 2}=-1.389396766$ ) and Calcyon (dopamine receptor D1 interacting protein, 219896_at, $P=4.12902 \mathrm{E}-06$, $\left.\mathrm{DE}_{\log 2}=-2.049585553\right)$. NSF binds the AMPA receptor GluR2 subunit and acts to disrupt GluR2-PICK1 interactions [28]; it may play a role in AMPA receptor trafficking 

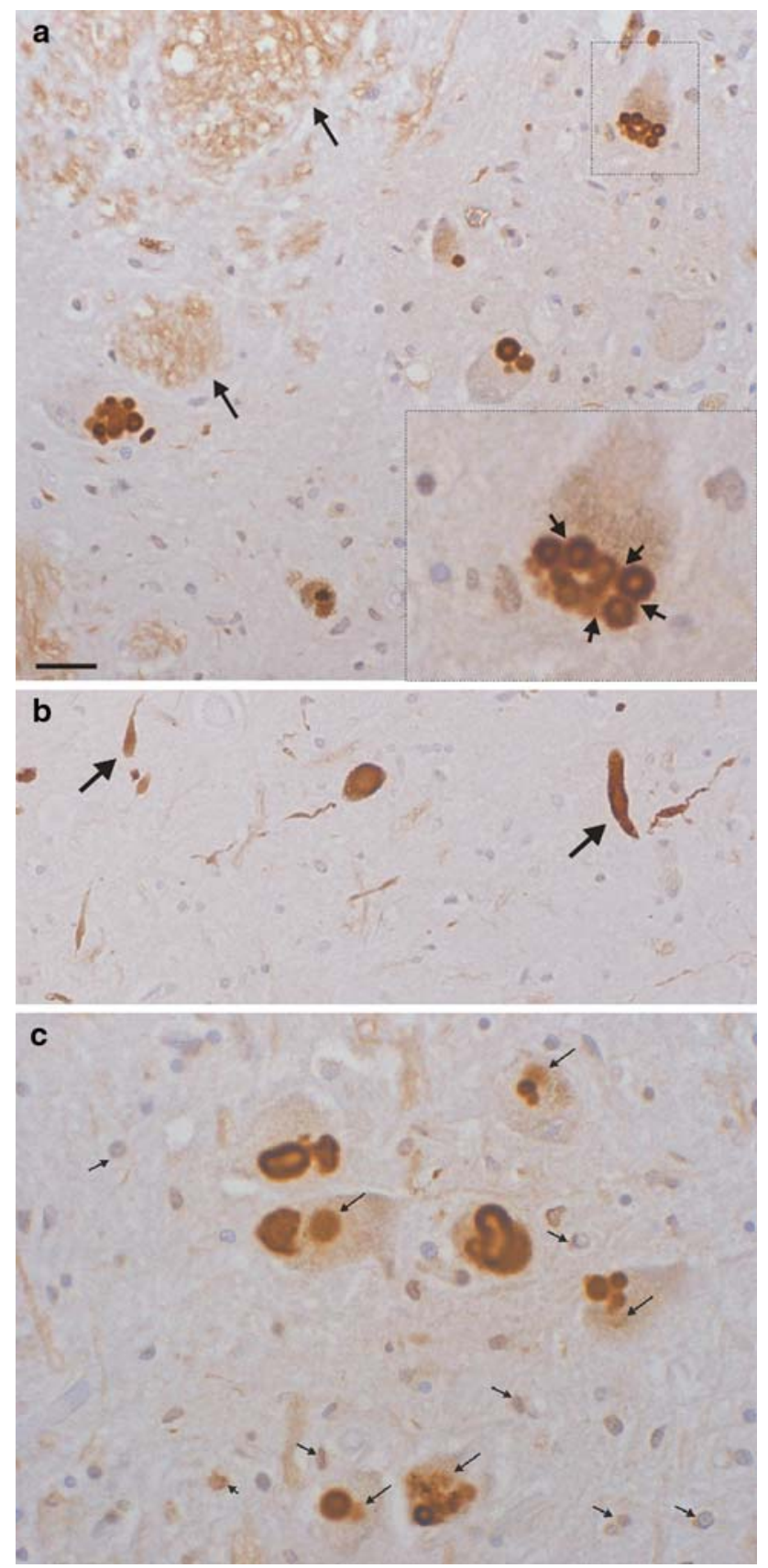

Fig. 3 Morphology of neuronal pentraxin II immunoreactive deposits in the Parkinsonian substantia nigra. a Lewy bodies are labelled which often show a crennelated surface (also see c). Arrows of the inset point to NPTX2 labelled connections/"bridges" between small Lewy bodies. Cross-sectioned axon bundles in white matter are numerous (longer arrows). b Arrows point to Lewy neurites in the substantia nigra. c NPTX2 is present in pre-Lewy bodies and glial cells. Labelling can be seen inside the cytoplasm of nerve cells in the neighbourhood and often directly attached to mature Lewy bodies (long arrows). Note the crenellated surface of compacted NPTX2 deposits (centre and Lewy body at the bottom of the figure) due to hair-like projections, which radiate into the cytoplasm. NPTX2 immunoreactivity is also present in glial cells. A subset of astrocytes as well as possibly microglia appear to be labelled (small arrows). The short arrow in the lower left points to what appears to be nuclear immunoreactivity. Scale bars: 40 (a), 50 (b) and $30($ c) $\mu \mathrm{m}$, respectively
Table 1 Correlation analysis for NPTX2 microarray expression data and the corresponding semi-quantitative immunocytochemical ratings for eight of the PD cases

\begin{tabular}{lll}
\hline $\begin{array}{l}\text { Case } \\
\text { number }\end{array}$ & $\begin{array}{l}\text { Expression } \\
\text { NPTX2 mRNA }(\log 2)\end{array}$ & $\begin{array}{l}\text { Semi-quantitative } \\
\text { ratings NPTX2 IR in SN }\end{array}$ \\
\hline 2 & 7.278105587 & 1 \\
3 & 9.800657274 & 3 \\
6 & 8.739679364 & 1.5 \\
7 & 8.44508108 & 1.5 \\
9 & 3.418203444 & 0.5 \\
12 & 7.980139578 & 1 \\
14 & 8.965264821 & 2 \\
15 & 6.414495823 & 1 \\
\hline
\end{tabular}

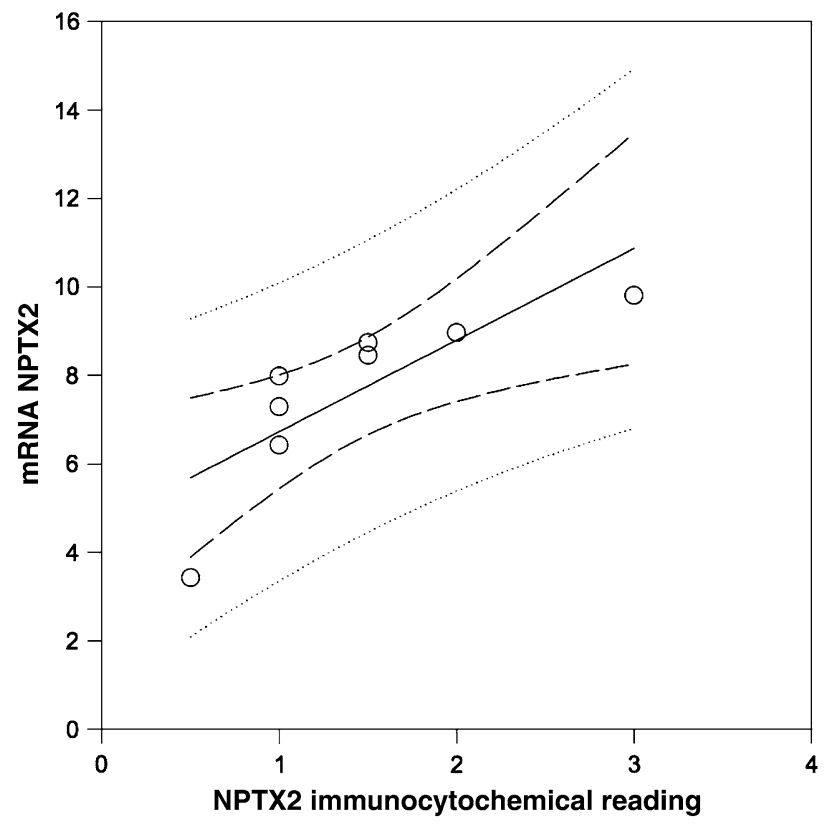

and stabilization. Calcyon is co-expressed in a number of D1 dopamine receptor-positive neurons in brain and, like D1 dopamine receptors, is found in dendritic spines [16]. We also found NPTX2 in glial cells possibly including microglia. However, staining of glia was only present in a subpopulation of cells while neuronal cell processes appeared to be consistently labelled. Why exactly NPTX2 accumulates in Lewy bodies remains unclear at present. However, it is conceivable that NPTX2 is involved in the uptake of synaptic material. Synaptic dysfunction in PD is very significant as suggested by our microarray experiments (unpublished data). The presence of NPTX2 in only a subset of Lewy bodies and its association with their "crenellated surface" shown in Fig. 3 as well as NPTX2 immunoreactive "bridges" between Lewy bodies might indicate a role of NPTX2 in Lewy body growth.

Many PD patients also show non-motor symptoms such as hallucinations, depression, autonomic dysfunction and 


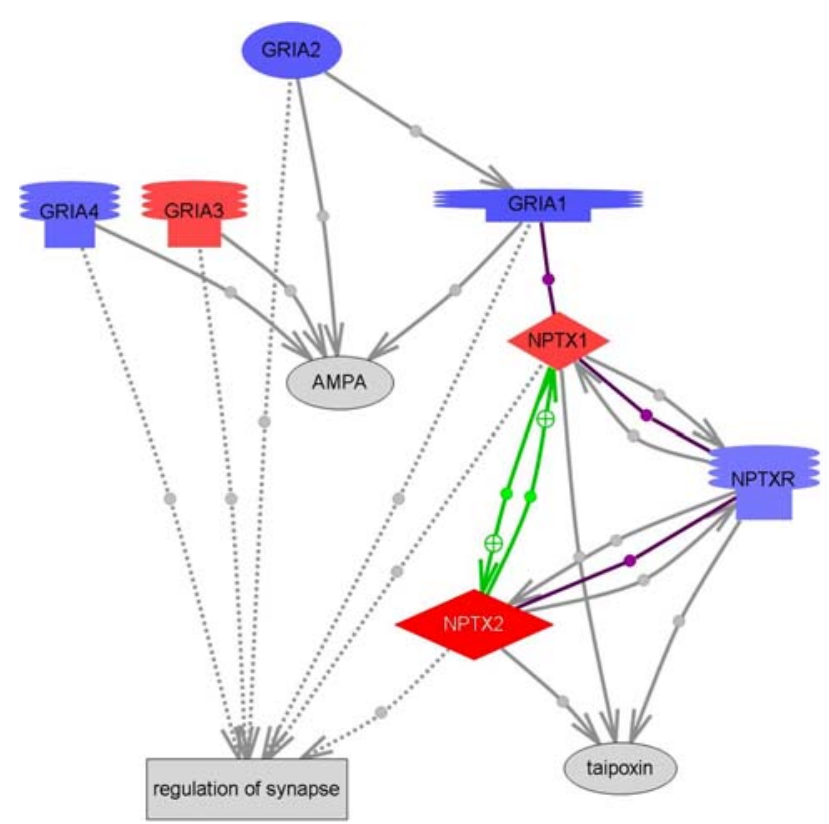

Fig. 4 NPTX2 pathway obtained by means of the Resnet 5.0 eukaryotic database of molecular interactions (Ariadne) overlayed with microarray expression values. Changes of PD relative to normal are indicated in red (increased in PD) and blue (downregulated), respectively. Abbreviations: AMPA alpha-amino-3-hydroxy-5-methyl-4-isoxazolepropionic acid; GRIAl glutamate receptor, ionotropic, AMPA 1; GRIA2 glutamate receptor, ionotropic, AMPA 2; GRIA3 glutamate receptor, ionotrophic, AMPA 3; GRIA4 glutamate receptor, ionotrophic, AMPA 4; NPTX1 neuronal pentraxin I; NPTX2 neuronal pentraxin II; NPTXR neuronal pentraxin receptor. Green line, regulation; purple line, binding. The dotted grey lines connect to a cell process whereas the solid lines connect to a molecule. The grey colour indicates that the effect and/or mechanism are unknown. References for all protein interactions shown are provided in the Electronic Supplement to this figure

sleep-disturbances. All these symptoms are present in narcolepsy where there is loss of orexin (hypocretin) producing nerve cells in the hypothalamus [2,30]. It is noteworthy therefore that the same population of neurons shows massive cell loss in PD as shown recently $[9,30]$. In addition, Thannickal et al. observed a significant correlation with the clinical stage of PD [30]. It is intriguing that orexin neurons of the hypothalamus demonstrate robust expression of Narp in the rat [27].

The relationship between NPTX2 and cell death deserves further study. All subunits of the NMDA, AMPA and kainate classes of glutamate receptors and a set of NMDA receptor-associated intracellular proteins are expressed in the human substantia nigra pars compacta, and may play a role in glutamate regulation of dopaminergic activity and/or release [21]. AMPA in turn has been discovered to be a mediator of selective non-apoptotic cell death in dopaminergic neurons [4]. The latter differs ultrastructurally from both neuronal apoptosis and typical excitotoxicity. Importantly, this mode of cell death is independent of caspase activity and does not have the morphological appearance of classical apoptosis. The findings are thus in keeping with our old postulate that nigral neurons are likely to die of a mechanism other than classical apoptosis and that aposklesis ("withering") may be a more appropriate term $[12,13]$. The involvement of NPTX2 in cell death mechanisms is additionally supported by studies on the effect of ceramide, which activates TNF-pathways [4]. Therefore, NPTX2/Narp has to be considered a new important gene in PD dysregulation which may be linked to both motor dysfunction caused by nigral dopaminergic nerve cell death in the midbrain and dementia due to synaptic changes in the cerebral cortex.

Acknowledgments We are most grateful to the brain donors and their relatives for providing tissue to the UK Parkinson's Disease Society Tissue Bank, funded by the Parkinson's disease Society of the UK, registered charity 948776 . We would like to thank Maurice R. Elphick for provision of the microscope and colour camera for photography of the in situ hybridization material. We would also like to thank the donors of the UK Multiple Sclerosis Society Tissue Bank.

\section{References}

1. Bjartmar L, Huberman AD, Ullian EM, Renteria RC, Liu X, Xu W, Prezioso J, Susman MW, Stellwagen D, Stokes CC, Cho R, Worley P, Malenka RC, Ball S, Peachey NS, Copenhagen D, Chapman B, Nakamoto M, Barres BA, Perin MS (2006) Neuronal pentraxins mediate synaptic refinement in the developing visual system. J Neurosci 26:6269-6281

2. Blouin AM, Thannickal TC, Worley PF, Baraban JM, Reti IM, Siegel JM (2005) Narp immunostaining of human hypocretin (orexin) neurons: loss in narcolepsy. Neurology 65:1189-1192

3. Crocker A, Espana RA, Papadopoulou M, Saper CB, Faraco J, Sakurai T, Honda M, Mignot E, Scammell TE (2005) Concomitant loss of dynorphin, NARP, and orexin in narcolepsy. Neurology 65:1184-1188

4. Decraene C, Brugg B, Ruberg M, Eveno E, Matingou C, Tahi F, Mariani J, Auffray C, Pietu G (2002) Identification of genes involved in ceramide-dependent neuronal apoptosis using cDNA arrays. Genome Biol 3:RESEARCH0042

5. Dorsey DA, Masco DH, Dikranian K, Hyrc K, Masciotra L, Faddis B, Soriano M, Gru AA, Goldberg MP, de Erausquin GA (2006) Ultrastructural characterization of alpha-amino-3-hydroxy-5-methyl-4-isoxazolepropionic acid-induced cell death in embryonic dopaminergic neurons. Apoptosis 11:535-544

6. Duke DC, Moran LB, Kalaitzakis ME, Deprez M, Dexter DT, Pearce RK, Graeber MB (2006) Transcriptome analysis reveals link between proteasomal and mitochondrial pathways in Parkinson's disease. Neurogenetics 7:139-148

7. Fong DK, Craig AM (1999) The Narp hypothesis? Neuron 23:195-197

8. Fox MA, Umemori H (2006) Seeking long-term relationship: axon and target communicate to organize synaptic differentiation. J Neurochem 97:1215-1231

9. Fronczek R, Overeem S, Lee SY, Hegeman IM, van Pelt J, van Duinen SG, Lammers GJ, Swaab DF (2007) Hypocretin (orexin) loss in Parkinson's disease. Brain 130:1577-1585

10. Fukumoto M, Iwata M, Shinbori C, Hazama GI, Shirayama Y, Kawahara R (2005) Effects of acute administration of methamphetamine on Narp mRNA in rat brain. Addict Biol 10:257-259 
11. Goodman AR, Cardozo T, Abagyan R, Altmeyer A, Wisniewski HG, Vilcek J (1996) Long pentraxins: an emerging group of proteins with diverse functions. Cytokine Growth Factor Rev 7:191202

12. Graeber MB, Grasbon-Frodl E, Abell-Aleff P, Kosel S (1999) Nigral neurons are likely to die of a mechanism other than classical apoptosis in Parkinson's disease. Parkinsonism Relat Disord 5:187-192

13. Graeber MB, Moran LB (2002) Mechanisms of cell death in neurodegenerative diseases: fashion, fiction, and facts. Brain Pathol 12:385-390

14. Hsu YC, Perin MS (1995) Human neuronal pentraxin II (NPTX2): conservation, genomic structure, and chromosomal localization. Genomics 28:220-227

15. Kirkpatrick LL, Matzuk MM, Dodds DC, Perin MS (2000) Biochemical interactions of the neuronal pentraxins. Neuronal pentraxin (NP) receptor binds to taipoxin and taipoxin-associated calcium-binding protein 49 via NP1 and NP2. J Biol Chem 275:17786-17792

16. Lezcano N, Mrzljak L, Levenson R, Bergson C (2006) Retraction. Science 314:1681

17. McAllister AK (2000) Cellular and molecular mechanisms of dendrite growth. Cereb Cortex 10:963-973

18. Michael GJ, Averill S, Nitkunan A, Rattray M, Bennett DL, Yan Q, Priestley JV (1997) Nerve growth factor treatment increases brain-derived neurotrophic factor selectively in TrkA-expressing dorsal root ganglion cells and in their central terminations within the spinal cord. J Neurosci 17:8476-8490

19. Moran LB, Duke DC, Deprez M, Dexter DT, Pearce RK, Graeber MB (2006) Whole genome expression profiling of the medial and lateral substantia nigra in Parkinson's disease. Neurogenetics 7:1-11

20. Moran LB, Croisier E, Duke DC, Kalaitzakis ME, Roncaroli F, Deprez M, Dexter DT, Pearce RK, Graeber MB (2007) Analysis of alpha-synuclein, dopamine and parkin pathways in neuropathologically confirmed parkinsonian nigra. Acta Neuropathol (Berlin) 113:253-263

21. Mueller HT, Haroutunian V, Davis KL, Meador-Woodruff JH (2004) Expression of the ionotropic glutamate receptor subunits and NMDA receptor-associated intracellular proteins in the substantia nigra in schizophrenia. Brain Res Mol Brain Res 121:60 69

22. O'Brien RJ, Xu D, Petralia RS, Steward O, Huganir RL, Worley P (1999) Synaptic clustering of AMPA receptors by the extracellular immediate-early gene product Narp. Neuron 23:309-323

23. O'Brien R, Xu D, Mi R, Tang X, Hopf C, Worley P (2002) Synaptically targeted narp plays an essential role in the aggregation of AMPA receptors at excitatory synapses in cultured spinal neurons. J Neurosci 22:4487-4498

24. Parkkinen L, Kauppinen T, Pirttila T, Autere JM, Alafuzoff I (2005) Alpha-synuclein pathology does not predict extrapyramidal symptoms or dementia. Ann Neurol 57:82-91

25. Peirson SN, Butler JN, Foster RG (2003) Experimental validation of novel and conventional approaches to quantitative real-time PCR data analysis. Nucleic Acids Res 31:e73

26. Reti IM, Baraban JM (2003) Opiate withdrawal induces Narp in the extended amygdale. Neuropsyopharmacol 28:1606-1613

27. Reti IM, R Reddy R, Worley PF, Baraban JM (2002) Selective expression of Narp, a secreted neuronal pentraxin, in orexin neurons. J Neurochem 82:1561-1565

28. Steinberg JP, Huganir RL, Linden DJ (2004) N-ethylmaleimidesensitive factor is required for the synaptic incorporation and removal of AMPA receptors during cerebellar long-term depression. Proc Natl Acad Sci 101:18212-18216

29. Storr HL, Clark AJ, Priestley JV, Michael GJ (2005) Identification of the sites of expression of triple A syndrome mRNA in the rat using in situ hybridisation. Neuroscience 131:113-123

30. Thannickal TC, Lai YY, Siegel JM (2007) Hypocretin (orexin) cell loss in Parkinson's disease. Brain 130:1586-1595

31. Tsui CC, Copeland NG, Gilbert DJ, Jenkins NA, Barnes C, Worley PF (1996) Narp, a novel member of the pentraxin family, promotes neurite outgrowth and is dynamically regulated by neuronal activity. J Neurosci 16:2463-2478

32. Xu D, Hopf C, Reddy R, Cho RW, Guo L, Lanahan A, Petralia RS, Wenthold RJ, O'Brien RJ, Worley P (2003) Narp and NP1 form heterocomplexes that function in developmental and activitydependent synaptic plasticity. Neuron 39:513-528 